\title{
SINGULAR INTEGRALS AND FRACTIONAL POWERS OF OPERATORS
}

\author{
BY \\ MICHAEL J. FISHER( $\left.{ }^{1}\right)$
}

\begin{abstract}
Recently R. Wheeden studied a class of singular integral operators, the hypersingular integrals, as operators from $L_{p}^{\alpha}(H)$ to $L_{p}(H) ; L_{p}^{\alpha}(H)$ is the range of the $\alpha$ th order Bessel potential operator acting on $L_{p}(H)$ with the inherited norm. The purposes of the present paper are to extend the known results on hypersingular integrals to complex indices, to extend these results to operators defined over a real separable Hilbert space, and to use Komatsu's theory of fractional powers of operators to show that the hypersingular integral operator $G^{\alpha}$ is $\int_{H}\left(-A_{y}\right)^{\alpha} f d \mu(y)$ when $\operatorname{Im}(\alpha)$ $\neq 0$ or when $\operatorname{Re}(\alpha)$ is not a positive integer where $A_{y} g$ is the derivative of $g$ in the direction $y$. The case where $\operatorname{Im}(\alpha)=0$ and $\operatorname{Re}(\alpha)$ is a positive integer is treated in a sequel to the present paper.
\end{abstract}

1. Introduction. Let $E$ be $N$-dimensional Euclidean space and let $d x$ denote Lebesgue measure on $E$. The theory of Lebesgue spaces of differentiable functions $L_{p}^{\alpha}(E, d x)$ is well known; [2]. $L_{p}^{\alpha}(E, d x), 1<p<\infty, 0 \leqq \operatorname{Re} \alpha<\infty$, is the range of the Bessel potential operator, $J^{\alpha}$, acting on $L_{p}(E)$. When $\alpha$ is a positive integer, $n, L_{p}^{n}(E)$ is also the space of weakly differentiable functions with derivatives of order $k$, $0 \leqq k \leqq n$, in $L_{p}(E)$.

R. Wheeden [19], [20] has studied a class of linear operators, the hypersingular integral operators, which map $L_{p}^{\alpha}(E)$ into $L_{p}(E)$ continuously. Let $\Omega(y)$ be positively homogeneous of degree zero on $E$ and suppose that $\|\Omega\|_{1}=\int_{\Sigma}|\Omega(\omega)| d \omega<\infty$ when $\Sigma$ is the unit sphere in $E$ and $d \omega$ denotes normalized Lebesgue measure on $\Sigma$. Set

$$
T^{\alpha}(f)=\lim _{\varepsilon \rightarrow 0} \int_{\|y\|>\varepsilon} R_{k}(f, y) \frac{\Omega(y) d y}{\|y\|^{N+\alpha}}
$$

where $N$ is the dimension of $E$ and where

$$
R_{k}(f, y)=f(x+y)-\sum_{|\beta| \leqq k} \frac{D^{\beta} f}{\beta !} y^{\beta}
$$

and $k \leqq \alpha<k+1$. $T^{\alpha}$ is a hypersingular integral operator on $L_{p}^{\alpha}(E)$ to $L_{p}(E)$ and $\left\|T^{\alpha} J^{\alpha} f\right\|_{p} \leqq N(\alpha)\|\Omega\|_{1}$ where $N(\alpha)$ depends only on $N$ and $\alpha$. When $\alpha$ is a positive

Presented to the Society, August 28, 1970; received by the editors June 24, 1970 and, in revised form, December 28, 1970.

AMS 1970 subject classifications. Primary 44A25; Secondary 46E15, 46E35, 47D10, 28 A40.

Key words and phrases. Hypersingular integral, fractional powers of operators, Bessel potential, singular integral operator, Calderon-Zygmund operator.

(1) Research supported in part by the National Science Foundation grant NSF-GP-8839. 
integer, $k$, the additional assumption must be made that $\int_{\Sigma} p_{k}(\omega) \Omega(\omega) d \omega=0$ where $p_{k}(\omega)$ is any homogeneous polynomial in $\omega$ with degree $k$. Then at $\alpha=k, T^{\alpha}$ is similar to a Calderon-Zygmund operator and $T^{k}$ acts boundedly from $L_{p}^{k}(E)$ to $L_{p}(E)$. If $0<\alpha<1$,

$$
T^{\alpha} f=\lim _{\varepsilon \rightarrow 0} \int_{\|y\|>\varepsilon}[f(x+y)-f(x)] \frac{\Omega(y)}{\|y\|^{N+\alpha}} d y
$$

this case is especially important and interesting when $\Omega(y) \equiv 1$, since then $T^{\alpha} J^{\alpha}$ is given by a measure.

The purposes of this paper are to study a new approach to the hypersingular integral operators, to extend the results of [19], [20] to complex indices, and to generalize these results to hypersingular integrals over a real separable Hilbert space. Thus we shall replace the finite-dimensional Euclidean space $E$ with a real separable Hilbert space $H$ which is generally infinite dimensional. We shall rely heavily upon the work of Komatsu [10], [11], [12], [13] on the theory of fractional powers of operators. In particular we shall observe that some of Komatsu's results can be used to prove Stein's basic lemma [17, Lemma 4] which asserts that $T^{\alpha} J^{\alpha}$ is given by convolution with a measure when $\Omega(\omega) \equiv 1$. In addition, Komatsu's results will be useful in streamlining the study of the general hypersingular integral.

Throughout this paper $K(\alpha), M(\alpha), N(\alpha)>0, K(\alpha, p), M(\alpha, p), N(\alpha, p)>0$ are constants which depend only on the parameters shown and which may vary in value with the occasion of their use. If $T$ is a closed densely defined operator on a Banach space $X, D(T)$ denotes the domain of $T$ and $R(T)$ denotes the range of $T$.

\section{Preliminaries.}

a. The normal distribution on Hilbert space. To minimize the discussion of measure theory on Hilbert space we refer the reader to the papers [7], [9] of L. Gross and [15], [16] of I. E. Segal.

Definition (Segal). A weak distribution on a real Hilbert space, $H$, is an equivalence class of linear maps, $F$, from the conjugate space $H^{*}$ of $H$ to real valued measurable functions (modulo null functions) on a probability space (depending on $F$ ). Two such maps, $F$ and $F^{\prime}$, are equivalent if for any finite set of vectors $y_{1}, \ldots, y_{k}$ in $H^{*}, F\left(y_{1}\right), \ldots, F\left(y_{k}\right)$ and $F^{\prime}\left(y_{1}\right), \ldots, F^{\prime}\left(y_{k}\right)$ have the same joint distribution in $k$-space. A weak distribution is continuous if a representative is a continuous linear map (the range space has the topology of convergence in measure).

In what follows we shall be most interested in the normal distribution with variance parameter $c / 2$. This distribution is uniquely determined by the following properties: for any $y$ in $H^{*}, F(y)$ is normally distributed with mean zero and variance $(c / 2)\|y\|^{2} ; F$ maps orthogonal vectors to independent random variables; $F$, a representative for the normal distribution, is continuous. There is an essentially unique (up to expectation preserving isomorphism) probability space $(S, \Sigma, \mu)$ 
and a continuous linear map $F$ from $H^{*}$ to the real valued measurable functions on $(S, \Sigma, \mu)$ (modulo null functions) such that $F$ is a representative of the normal distribution. $\Sigma$ has no proper sub- $\sigma$-field with respect to which all of the $F(y)$, $y \in H^{*}$, are measurable. The measurable functions on $H$ are defined to be the measurable functions on $(S, \Sigma, \mu) . L_{p}\left(H, n_{c}\right)=L_{p}(S, \Sigma, \mu)$ by definition. When $c=2$, we let $n=n_{2}$ and $L_{p}(H)=L_{p}\left(H, n_{2}\right)$. The expectation, $E(f)$, of a measurable function $f$ is $E(f)=\int_{S} f d \mu$.

A function $f(x)$ on the points of $H$ is a tame function if there is a Baire function $g$ on a finite-dimensional Euclidean space $E_{k}$, and orthonormal vectors $h_{1}, \ldots, h_{k}$ in $H^{*}$ such that $f(x)=g\left(\left(x, h_{1}\right), \ldots,\left(x, h_{k}\right)\right)$. The span of the $h_{1}, \ldots, h_{k}$ in $H$ is called the base of $f$. If $F$ is a representative of the normal distribution and $f(x)$ $=g\left(\left(x, h_{1}\right), \ldots,\left(x, h_{k}\right)\right)$ is a tame function, then $f(s)=g\left(F\left(h_{1}\right)(s), \ldots, F\left(h_{k}\right)(s)\right)$ is a measurable function on $H$, and the expectation of $f$ is

$$
E(\tilde{f})=(\pi c)^{-k / 2} \int_{E_{k}} g(t) \exp \left(-c^{-1}\|t\|^{2}\right) d t
$$

where $k$ is the dimension of the base space of $f$. This equality holds in the sense that if either side exists and is finite, then so does the other side and the two are equal.

Several very useful representatives of the normal distribution are known. Of these the one in which we shall be most interested is the mapping studied by Gross [9] from $H^{*}$ to Borel measurable functions (modulo null functions) on an abstract Wiener space. We adopt the notation and terminology of [9]. Let $B$ be a one-one Hilbert-Schmidt operator on a real separable Hilbert space $H$. Then $|x|_{1}=\|B x\|$ is a measurable norm on $H$. Let $H_{B}$ denote the completion of $H$ in this norm. Let $\mathscr{S}$ denote the $\sigma$-field generated by the closed subsets of $H_{B}$. The normal distribution $n_{c}$ induces a Borel probability measure $N_{c}$ on $H_{B}$ such that the extension of the identity map on $H_{B}^{*}\left(\subset H^{*}\right)$, regarded as a densely defined map on $H^{*}$ to measurable functions on $\left(H_{B}, \mathscr{S}, N_{c}\right)$, to $H^{*}$ is a representative of the normal distribution on $H$. Continuous functions $f$ on $H_{B}$ are measurable functions on $H$ and if $g$ denotes the restriction of $f$ to $H$ and if $\mathscr{F}$ denotes the directed set (ordered by inclusion of the ranges) of finite-dimensional projections on $H$, the net $\{\tilde{g}(Q x): Q \in \mathscr{F}\}$ of measurable tame functions converges in measure to $f$ as $Q$ tends strongly to the identity through $\mathscr{F}$.

Let $N_{c}$ be as above, and regard $B$ as an isometry from $H_{B}$ to $H$. Then $N_{c} \circ B^{-1}$ is a Borel measure on $H$; this measure is usually denoted by $n_{c} \circ B^{-1}$; [8], [9]. If $f$ is a bounded continuous function from $H$ to a Banach space $X, \int_{H} f(x) d n_{c} \circ B^{-1}(x)$ $=\int_{H_{B}} f(B y) d N_{c}(y)=E(\tilde{f} \circ B)$. If $f, g$, and $f g$ are absolutely integrable tame functions on $H, \tilde{f} g=\tilde{f} \tilde{g}$, $(a f+g)^{\sim}=a \tilde{f}+\tilde{g}$ for constants $a$, and if $f \leqq g$ (a.e.) on $H$, then $\tilde{f} \leqq \tilde{g}$ (a.e.); we shall use these properties often. Gross [7], [9] has studied other functions on the points of $H$ which give rise to measurable functions on $H$. If $C$ is a Hilbert-Schmidt operator on $H,\|C x\|$ determines a measurable function 
$\|C(\cdot)\|^{\sim}$ on $H$ by $\|C(\cdot)\|^{\sim}=\lim \left\{\|C Q x\|^{\sim}: Q \in \mathscr{F}\right\}$ where $\mathscr{F}$ is as above and $Q$ tends strongly to the identity through $\mathscr{F}$. If $f$ is a bounded continuous function on $H$, then $f(C x)$ determines a measurable function on $H$ when $C$ is a Hilbert-Schmidt operator. Such elementary functions as $\|x\|, \exp \left(-\|x\|^{2}\right)$ and $\exp (i\|x\|)$ fail to determine measurable functions on $H$. We refer to [7] for further discussion of these points.

b. The Poisson integral. Let $H$ be a real separable Hilbert space. Let $L_{p}(H)$, $1<p<\infty$, denote the Banach space of $p$-power integrable functions with respect to the weak normal distribution (with variance parameter 1 , centered at the origin) on $H$. Let $y \rightarrow T_{y}$ denote the regular representation of the additive group of $H$ by isometries on $L_{p}(H)$; if $f$ is a bounded tame function,

$$
\left(T_{y} f\right)(x)=f(x-y) \exp \left[\frac{(x, y)}{p}-\frac{\|y\|^{2}}{2 p}\right] .
$$

The $T_{y}$ are strongly continuous and play the role of the "translation operators" on $H$; [4]. If $\mu$ is a finite Borel measure on $H, T(f)=\int_{H} T_{y} f d \mu(y)$ is a bounded operator on $L_{p}(H)$ with norm at most $\|\mu\|$. If $n_{t}$ denotes the normal distribution on $H$ with variance parameter $t / 2$, and if $B$ is a Hilbert-Schmidt operator on $H$, then $n_{t} \circ B^{-1}$ is a Borel probability measure on $H$; [8]. Let

$$
H_{t}(f)=\int_{H} T_{y}(f) d n_{t} \circ B^{-1}(y)
$$

and

$$
P_{z}(f)=\int_{0}^{\infty} H_{t}(f) N_{t}(z) d t / t
$$

where $N_{t}(z)=(\pi t)^{-1 / 2} z \exp \left(-t^{-1} z^{2}\right) . P_{z}(f)$ is the Poisson integral of $f . H_{t}(f)$ and $P_{z}(f)$ were studied in [5]. We shall recall some of the properties of these operators; the proofs appear in [5].

P-1. $H_{t}$ and $P_{z}$ are strongly continuous contraction semigroups on $L_{p}(H)$.

P-2. There is a unique Borel probability measure $p_{z}$ on $H$ such that $P_{z}(f)$ $=\int_{H} T_{y} f d p_{z}(y)$.

P-3. If $a=\left(a_{1}, \ldots, a_{n}\right)$ is a multi-index with $|a|=\sum_{i=1}^{n} a_{i}$, if $A_{h}$ is the infinitesimal generator of the translation semigroup $T_{t B h}, t>0$, and if $A^{a}=A_{h_{1}}^{a_{1}} \cdots A_{h_{n}}^{a_{n}}$, then

$$
A^{a} H_{t}(f)=\int_{H_{B}} T_{b y} f C^{a}(1)(y) d n_{t}(y)
$$

where $C^{a}=C_{h_{1}}^{a_{1}} \ldots C_{h_{n}}^{a_{n}}$ and where $C_{h}$ is the infinitesimal generator of $T_{s h}, s>0$, acting on $L_{1}\left(H, n_{t}\right)$. Thus if $t>0, A^{a} H_{t}$ is a bounded operator on $L_{p}(H)$ and $\left\|A^{a} H_{t}\right\| \leqq N(a)\left\|h_{1}\right\|^{a_{1}} \cdots\left\|h_{n}\right\|^{a_{n} t^{-|a| / 2}}$. 
P-4. $P_{z}(f)$ is infinitely differentiable with respect to $z$ and with respect to the space variables;

$$
A^{a} P_{z}(f)=\int_{0}^{\infty} A^{a} H_{t}(f) N_{t}(z) d t / t
$$

and

$$
\frac{d^{n}}{d x^{n}} P_{x}(f)=\int_{0}^{\infty} H_{t}(f) \frac{d^{n}}{d x^{n}} N_{t}(x) d t / t .
$$

We shall use the infinitesimal generators $A_{h}$ of $T_{t B h}$ as the Hilbert space analogues of the directional derivatives $D_{h}$ on finite-dimensional Euclidean space.

c. Komatsu powers of operators. Early work on the theory of fractional powers of operators is surveyed in [21]. Komatsu [10], [11], [12], [13] has developed an extensive theory of fractional powers of operators. In [10], [11] it is assumed that $A$ is a linear operator (not necessarily densely defined) such that the negative half-line is in the resolvent set of $A$ and $\left\|t(t+A)^{-1}\right\| \leqq M$ for all $t>0$. $A^{\alpha}$ is defined for all complex $\alpha$ in $\$ 4$ of [10]. For our purposes it will be sufficient to recall some of Komatsu's results for the case when $(-A)$ generates a bounded, strongly continuous semigroup on a reflexive Banach space $X$.

$\mathrm{K}-1$. If $0<\operatorname{Re} \alpha<1$,

$$
A^{\alpha} x=\frac{\sin \pi \alpha}{\pi} \int_{0}^{\infty} t^{\alpha-1} A(t+A)^{-1} x d t
$$

when $x \in D(A)$, the domain of $A ;[10$, p. 299].

$\mathrm{K}-2$. If $0<\operatorname{Re} \alpha<\sigma<n, n$ a positive integer, then

$$
A^{\alpha} x=\frac{\Gamma(m)}{\Gamma(\alpha) \Gamma(m-\alpha)} \int_{0}^{\infty} t^{\alpha-1}\left(A(t+A)^{-1}\right)^{m} x d t
$$

for $x \in D\left(A^{N}\right)$ when $N>m>n$; [11, p. 292].

$\mathrm{K}-3$. If $(-A)$ generates a bounded strongly continuous semigroup $T_{t}$ on $X$, then if $x \in D(A)$ and $0<\operatorname{Re} \alpha<\sigma<1$,

$$
A^{\alpha} x=\Gamma(-\alpha)^{-1} \int_{0^{+}}^{\infty}\left(T_{t} x-x\right) t^{-\alpha-1} d t ; \quad[10, \text { p. 325]. }
$$

More formally, K-1 and K-3 define an operator $A_{\sigma}^{\alpha}$ on a subspace $D^{\sigma}$ of $X ; D^{\sigma}$ is defined in [10]. If $A_{+}^{\alpha}$ denotes the smallest closed extension of $A_{\sigma}^{\alpha}$, whose existence is proved in [10, Proposition 4.1], then $A^{\alpha}=A_{+}^{\alpha}$. Similarly K-2 defines an operator on a natural subspace of $X$ and its smallest closed extension is $A_{+}^{\alpha}=A^{\alpha}$ as is shown in [11]. When $\operatorname{Re} \alpha<0, A_{-\sigma}^{\alpha}$ is defined by equation 4.10 of [10, p. 304] and $A_{-\sigma}^{\alpha}$ is shown to have a smallest closed extension $A_{-}^{\alpha}$ which is independent of $\sigma$. When $\operatorname{Re} \alpha=0, A^{\alpha} x$ is defined by equation 4.11 of $\left[10\right.$, p. 305] for $x \in D^{\sigma} \cap R^{x}$. There is the important 
$\mathrm{K}-4$. For any complex $\alpha, A_{\sigma \tau}^{\alpha}$ has the smallest closed extension $A_{0}^{\alpha}$ which is independent of $\sigma$ and $\tau$ when $-\tau<\operatorname{Re} \alpha<\sigma$. If $\operatorname{Re} \alpha>0, A_{0}^{\alpha}=A_{+}^{\alpha}$ on $D\left(A_{+}^{\alpha}\right)$ $\cap \mathrm{Cl} R(A)$ and if $\operatorname{Re} \alpha<0, A_{0}^{\alpha}=A_{-}^{\alpha}$.

If $A$ has a bounded inverse, $R^{\sigma}=X$ and $A_{-}^{\alpha}$ is everywhere defined and analytic if $\operatorname{Re} \alpha<0$. If $x \in D^{\sigma}, A^{\alpha}$ is analytic in $\operatorname{Re} \alpha<\sigma$. If $-(n+1)<\operatorname{Re} \alpha<0$,

$$
A_{-}^{\alpha}=\left(\frac{-\sin \pi \alpha}{\pi}\right) \frac{n !}{(\alpha+1) \cdots(\alpha+n)} \int_{0}^{\infty} t^{\alpha+n}(t+A)^{-n-1} d t
$$

and

K-5. When $A$ has a bounded inverse, if $\operatorname{Re} \alpha>0$, then $A_{+}^{\alpha}=A_{0}^{\alpha}$ is the inverse of $A_{0}^{-\alpha}=A_{-}^{-\alpha} ; D\left(A_{+}^{\alpha}\right)$ is contained in $R\left(A_{-}^{-\alpha}\right)$. See $\S 5$ of [10].

K-6. (i) If $\operatorname{Re} \alpha \operatorname{Re} \beta>0$, then $A_{ \pm}^{\alpha} A_{ \pm}^{\beta}=A_{ \pm}^{\alpha+\beta}$ in the sense of the product of operators. (ii) If $\alpha$ and $\beta$ are any complex numbers, then $\left[A_{0}^{\alpha} A_{0}^{\beta}\right]_{C}=A_{0}^{\alpha+\beta}$ where $[T]_{C}$ denotes the smallest closed extension of $T$. (iii) If $A$ has a bounded inverse and if $\operatorname{Re} \alpha>0$, then $A_{0}^{\alpha} A_{0}^{\beta}=A_{0}^{\alpha+\beta}$. See $\S 7$ of [10].

From the assumption that $\left\|t(t+A)^{-1}\right\| \leqq M$ for $t>0$ and the resolvent equation it follows that $(t+A)^{-1}$ exists for $t$ in the sector $|\arg t|<\operatorname{Arc} \sin \left(M^{-1}\right)$ and that $t(t+A)^{-1}$ is bounded on each ray of this sector. Let

$$
M(\Theta)=\sup \left\{\left\|t(t+A)^{-1}\right\|:|\arg t|=\Theta\right\}, \quad \Theta>0
$$

$M(\Theta)$ is an increasing function of $\Theta$. An operator $A$ is said to be of type $(\omega, M(\Theta))$, $0 \leqq \omega<\pi$, if $A$ is closed, densely defined; the resolvent set of $(-A)$ contains the sector $|\arg t|<\pi-\omega$, and $\sup \left\{\left\|t(t+A)^{-1}\right\|:|\arg t|=\Theta\right\} \leqq M(\Theta)<\infty$ holds for all $0 \leqq \Theta<\pi-\omega$. An operator $A$ is of type $(\omega, M(\Theta))$ for an $\omega<\pi / 2$ if and only if $(-A)$ generates a semigroup $T_{t}$ which has an analytic extension to the sector $|\arg t|$ $<\pi / 2-\omega$ such that the extension is uniformly bounded on each sector $|\arg t|$ $\leqq \pi / 2-\omega-\varepsilon, \varepsilon>0$.

$\mathrm{K}-7$. If $A$ is an operator of type $(\omega, M(\Theta))$ and $0<\alpha \omega<\pi / 2$, then $\left(-A_{+}^{\alpha}\right)$ is the generator of a strongly continuous semigroup $\exp \left(-t A_{+}^{\alpha}\right)$ which is analytic in the sector $|\arg t| \leqq \pi / 2-\alpha \omega$ and uniformly bounded on each smaller sector $|\arg t|$ $\leqq \pi / 2-\alpha \omega-\varepsilon, \varepsilon>0$. See $\S 10$ of [10].

K-8. Let $A$ be of type $(\omega, M(\Theta))$. Then $\left(A_{+}^{\alpha}\right)^{\beta}=A_{+}^{\alpha \beta}$ if $0<\alpha<\pi / \omega$ and $\operatorname{Re} \beta>0$.

K-9. If $0<\alpha<1$ and if $T_{t}=\exp (-t A), T_{t}^{\alpha} x=\exp \left(-t A^{\alpha}\right) x=\int_{0}^{\infty} T_{s} x N(\alpha, t, s) d s$ where $N(\alpha, t, s)=(2 \pi i)^{-1} \int_{\sigma-i \infty}^{\sigma+i \infty} \exp \left(u s-t u^{\alpha}\right) d u ;[21]$.

It is worth noting in connection with the discussion preceding $\mathrm{K}-8$ and in connection with K-8 that the Poisson integral $P_{z}$ extends to an analytic semigroup in every sector $|\arg z|<\pi / 4-\varepsilon, 0<\varepsilon<\pi / 4$, and the extension is bounded in each sector of this type; this follows from the fact that $N_{t}(z)$ is analytic in $\operatorname{Re} z>0$ and the fact that the integral $P_{z}(f)=\int_{0}^{\infty} H_{t}(f) N_{t}(z) d t / t$ converges uniformly in $|\arg z|<\pi / 4-\varepsilon$ for $\varepsilon>0$. If $H_{t}=\exp (-t A)$, then $P_{z}=\exp (-z T)$ where $T=A^{1 / 2}$; see K-9 and [21]. 
When the Hilbert-Schmidt operator $B$ is one-one, the infinitesimal generator $(-T)$ of $P_{z}$ is a one-one operator on $L_{p}(H)$ and $R(T)$ is dense in $L_{p}(H)$.

d. Definitions and hypersingular integrals on $L_{2}(H)$. Let $\alpha=\beta+i \gamma, \operatorname{Re} \alpha>0$, be a complex number and let $\mu$ be a Borel measure (not necessarily bounded) on $H$ such that

$$
\int_{H}\|y\|^{\beta} d|\mu|(y)<\infty, \quad \beta=\operatorname{Re} \alpha .
$$

For any nonnegative integer $k$, if $k>0$ set

$$
R_{k}(f, y, t)=[(k-1) !]^{-1} \int_{0}^{t}(t-u)^{k-1}\left(T_{u B y} A_{y}^{k} f-A_{y}^{k} f\right) d u,
$$

and if $k=0$ set $R_{0}(f, y, t)=T_{t B y} f-f$ when $f \in D\left(A_{y}^{k}\right) \cap L_{p}(H)$ for all $y$ in $H$. If $k \leqq \operatorname{Re} \alpha<k+1$, set

$$
G^{\alpha}(f)=\int_{0^{+}}^{\infty} \int_{H} R_{k}(f, y, t) d \mu(y) t^{-\alpha-1} d t
$$

whenever this integral converges in $L_{p}(H) . G^{\alpha}(f)$ is a hypersingular integral operator. We shall make further assumptions on $\alpha$ later so that $G^{\alpha}$ is defined when $\operatorname{Re} \alpha$ $=n$ is a positive integer.

In this section we shall sketch the $L_{2}$-theory of hypersingular integral operators in order to motivate the more formal theory needed to prove our main theorems in $\S 4$.

Let $p=2$ and $P(y)$ be a polynomial on $H$. Set $W(P)(y)=\int_{H} P\left(2^{1 / 2} x+i y\right) d n(x)$ where $d n$ is the normal cylinder set measure with variance parameter 1 . Segal [15] has shown that $W(P)(y)$ extends to a unitary operator on $L_{2}(H) ; W$ is the Hilbert space analogue of the Fourier transform. In particular, $W\left(T_{y} f\right)=$ $\exp (i F(y) / 2) W(f)$ where $F$ is a representative of the normal distribution; hence $W\left(A_{y} f\right)=(i F(y) / 2) W(f)$ when $f \in D\left(A_{y}\right)$.

Consider the linear operator $J^{\alpha}$ on $L_{2}(H)$ defined by

$$
W\left(J^{\alpha} f\right)=\left(1+\left\|B^{*}(\cdot)\right\|\right)^{-\alpha} W(f)
$$

when $\operatorname{Re} \alpha \geqq 0$ and $B$ is the one-one Hilbert-Schmidt operator used to define $H_{t}$ and $P_{z} . J^{\alpha}$ is a bounded operator on $L_{2}(H)$. Some other properties of $J^{\alpha}$ are

1. $J^{\alpha} J^{\beta}=J^{\alpha+\beta}$ if $\operatorname{Re} \alpha, \operatorname{Re} \beta \geqq 0$.

2. $J^{\alpha}$ is strongly analytic in $\operatorname{Re} \alpha>0$.

3. $J^{\alpha}$ is one-one.

4. If $\operatorname{Re} \alpha \geqq 0$ and if $\beta=\left(\beta_{1}, \ldots, \beta_{n}\right)$ is a multi-index of complex numbers with $\operatorname{Re} \beta_{i} \geqq 0$, and $|\operatorname{Re} \beta| \leqq \operatorname{Re} \alpha$, then $A_{h}^{\beta} J^{\alpha}$ is a bounded operator on $L_{2}(H)$.

Each of these properties is easily verified.

THEOREM 1. If $\operatorname{Re} \alpha>0$ is not an integer, $G^{\alpha} J^{\alpha}$ is a bounded operator on $L_{2}(H)$ with $\left\|G^{\alpha} J^{\alpha}\right\| \leqq N(\alpha) \int_{H}\|y\|^{\beta} d|\mu|(y), \operatorname{Re} \alpha=\beta$. 
Proof. Let $k<\operatorname{Re} \alpha<k+1$ and $\alpha=k+\delta$. Then $t^{-k} R_{k}(f, y, t)=t^{-k} R_{k}(f, t y, 1)$, and since $A_{t y}^{k}=t^{k} A_{y}^{k}$,

$$
W\left(G^{\alpha} J^{\alpha} f\right)=\int_{0^{+}}^{\infty} \int_{H} t^{-k} W\left(R_{k}\left(J^{\alpha} f, y, t\right)\right) d \mu(y) t^{-\delta-1} d t,
$$

and

$$
W R_{k}\left(J^{\alpha} f, t y, 1\right)=[(k-1) !]^{-1} \int_{0}^{1}(1-u)^{k-1}(\exp (i t u F(B y) / 2)-1) d u \varphi(y),
$$

where $\varphi(y)=(i F(B y) / 2)^{k}\left(1+\left\|B^{*}(\cdot)\right\|\right)^{-\alpha}$. Since

$$
\begin{gathered}
\int_{0^{+}}^{\infty}[\exp (i t F(B y) / 2)-1] t^{-\delta-1} d t=\Gamma(-\delta)(-i F(B y) / 2)^{\delta}, \\
W\left(G^{\alpha} J^{\alpha} f\right)=M(\alpha) W(f) \int_{H}[-i F(B y)]^{\alpha}\left(1+\left\|B^{*}(\cdot)\right\|\right)^{-\alpha} d \mu(y) .
\end{gathered}
$$

Since $|\langle\xi, B y\rangle|\left(1+\left\|B^{*} \xi\right\|\right)^{-1} \leqq\|y\|$,

$$
\left\|G^{\alpha} J^{\alpha}\right\| \leqq N(\alpha) \int_{H}\|y\|^{\beta} d|\mu|(y)<\infty, \quad \beta=\operatorname{Re} \alpha .
$$

Note that $M(\alpha)=\Gamma(k)^{-1} \Gamma(-\delta) B(k, \delta+1)(-1)^{k} 2^{-\alpha}$ where $B(\cdot, \cdot)$ is the $\beta$-function.

The next theorem extends Theorem 1 to the case when $\operatorname{Re} \alpha=k$, a positive integer.

TheOREM 2. Let $\operatorname{Re}(\alpha)=k \geqq 1$ and suppose that if $p_{k}$ is a homogeneous polynomial of degree $k$ on $H$ then $\int_{H} p_{k}(y) d \mu(y)=0$. Then if $\operatorname{Im}(\alpha) \neq 0,\left\|G^{\alpha} J^{\alpha}\right\|$ $\leqq N(\alpha) \int_{H}\|y\|^{k} d|\mu|(y) . \operatorname{If} \operatorname{Im}(\alpha)=0,\left\|G^{\alpha} J^{\alpha}\right\| \leqq N(\alpha) \int_{H}\|y\|^{k}(1+|\log \|y\||) d|\mu|(y)$.

Proof. Proceed as in the proof of Theorem 1 to get

$$
W G^{\alpha} J^{\alpha} f=M(k) \int_{H} \int_{0^{+}}^{\infty}[\exp (i t F(B y))-1] t^{-1-i \gamma} d t F(B y)^{k} d \mu(y) W\left(J^{\alpha} f\right)
$$

where $\gamma=\operatorname{Im} \alpha$. Assume first that $\gamma \neq 0$. Since $\int_{H} F(B y)^{k} d \mu(y)=0$, Corollary 1 of [14] implies that the double integral above converges to a bounded function. Thus $\left\|G^{\alpha} J^{\alpha}\right\| \leqq N(\alpha) \int_{H}\|y\|^{k} d|\mu|(y)$.

When $\operatorname{Re} \alpha=k$ and $\operatorname{Im} \alpha=0, G^{\alpha} J^{\alpha}$ is a kind of Calderon-Zygmund operator; [1], [4]. Set $d \nu(y)=\left(1+\left\|B^{*}(\cdot)\right\|\right)^{-k} F(B y)^{k} d \mu(y)$, so we need only show that

$$
\Delta=\int_{H} \int_{0^{+}}^{\infty}[\exp (i t\langle\xi, B y\rangle)-1] d t / t d \nu(y)
$$

satisfies $|\Delta| \leqq N \int_{H}\|y\|^{k}(1+|\log \|y\||) d|\mu|(y)$. The integral $\Delta$ can be evaluated by the method of contour integration as in the proof of Theorem 5 (Lemma 5.3) of [4]; in fact $\Delta$ is the integral evaluated there. The value of $\Delta$ is

$$
\int_{H}\left\{i \pi / 2 \operatorname{sgn}\left\langle B^{*} \eta, y\right\rangle-\log \left|\left\langle B^{*} \eta, y\right\rangle\right|\right\} d \nu(y)
$$

where $\eta=\xi\|\xi\|^{-1}$. Thus $G^{k} J^{k}$ has the desired norm. 
REMARKS. It is interesting to note that $G^{k} J^{k}=T^{k} Z$ where $T^{k}$ has Wiener transform (or Fourier transform) $\left\|B^{*} x\right\|^{k}\left(1+\left\|B^{*} x\right\|\right)^{-k}$ and where $Z$ is a kind of Calderon-Zygmund operator. An analogue of the first statement of Theorem 2 holds when $\operatorname{Re}(\alpha)=0, \alpha \neq 0$. When $\alpha=0, G^{\alpha}$ is a Calderon-Zygmund operator if $\mu(H)=0$ and if $\int_{H}(1+|\log \|y\||) d|\mu|(y)<\infty$.

\section{Bessel potentials.}

Definition. If $f$ is in $L_{p}(H)$ and if $P_{t}(f)$ is the Poisson integral of $f$, then for $\operatorname{Re}(\alpha)>0$, the Bessel potential of $f$ of order $\alpha$ is

$$
J^{\alpha}(f)=\Gamma(\alpha)^{-1} \int_{0}^{\infty} P_{t}(f) t^{\alpha-1} e^{-t} d t .
$$

THEOREM 3. $J^{\alpha}$ enjoys the following properties:

1. $\left\|J^{\alpha}(f)\right\|_{p} \leqq \Gamma(\operatorname{Re} \alpha)\left|\Gamma(\alpha)^{-1}\right|\|f\|_{p}$.

2. $\lim \left\{J^{\alpha}(f):|\arg \alpha| \leqq \Theta<\pi / 2, \alpha \rightarrow 0\right\}=f$.

3. $J^{\alpha}$ is strongly analytic in $\operatorname{Re} \alpha>0$.

4. $J^{\alpha} J^{\beta}=J^{\alpha+\beta}$ if $\operatorname{Re} \alpha, \operatorname{Re} \beta>0$.

5. $J^{\alpha}$ is one-one on $L_{p}(H)$ if $\operatorname{Re} \alpha>0$.

6. $J^{\alpha}=\left(J^{1}\right)^{\alpha}$, the $\alpha$ th Komatsu power of $J^{1}$, if $\operatorname{Re} \alpha>0$.

7. The range of $J^{\alpha}, R\left(J^{\alpha}\right)$, is dense in $L_{p}(H)$.

8. $J^{i \gamma}$ is a strongly continuous group of bounded operators on $L_{p}(H)$ for real $\gamma$.

9. $J^{i \gamma}(f)=\lim \left\{J^{\varepsilon+i \gamma}(f): \varepsilon \rightarrow 0^{+}\right\}$for $f$ in $L_{p}(H)$.

Proof. (1) follows from Minkowski's integral inequality and the fact that $\left\|P_{t} f\right\|_{p} \leqq\|f\|_{p}$. If $\operatorname{Re} \alpha>0, J^{\alpha} f-f=\Gamma(\alpha)^{-1} \int_{0}^{\infty}\left[P_{t} f-f\right] t^{\alpha-1} e^{-t} d t$. Given $\varepsilon>0$, let $\delta>0$ be sufficiently small that $\left\|P_{t} f-f\right\|_{p}<\varepsilon$ for $0<t<\delta$. Choose $\eta>0$ such that $|\Gamma(\alpha)|^{-1} \int_{\delta}^{\infty} t^{\operatorname{Re} \alpha-1} e^{-t} d t<\varepsilon \Gamma(\operatorname{Re} \alpha)|\Gamma(\alpha)|^{-1}$ when $0<\operatorname{Re} \alpha<\eta$. Then $\left\|J^{\alpha} f-f\right\|_{p}$ $\leqq \varepsilon \Gamma(\operatorname{Re} \alpha)|\Gamma(\alpha)|^{-1}\left[1+2\|f\|_{p}\right]$. This proves (2) when $|\arg \alpha| \leqq \Theta<\pi / 2$, in which case $\Gamma(\operatorname{Re} \alpha)|\Gamma(\alpha)|^{-1} \leqq M(\Theta)$. (3) is a consequence of the analyticity of $\Gamma(a)^{-1} t^{\alpha-1}$ in $\operatorname{Re} \alpha>0$ for $t>0$ and the fact that $t^{\alpha-1} \log t e^{-t}$ is absolutely integrable for $\operatorname{Re} \alpha$ $>0$. To prove (4) let $\varphi_{\alpha}(t)=\Gamma(\alpha)^{-1} t^{\alpha-1} e^{-t}$ if $t>0$ and $\varphi_{\alpha}(t)=0$ if $t \leqq 0$. Then $J^{\alpha}(f)=\int_{-\infty}^{\infty} P_{t}(f) \varphi_{\alpha}(t) d t$ and $J^{\alpha} J^{\beta}(f)=\int_{-\infty}^{\infty} P_{t}(f) \varphi_{\alpha} * \varphi_{\beta}(t) d t$ where $\varphi_{\alpha} * \varphi_{\beta}$ is the convolution of $\varphi_{\alpha}$ and $\varphi_{\beta}$. An elementary integration and use of some basic identities for the $\Gamma$-function show that $\varphi_{\alpha} * \varphi_{\beta}=\varphi_{\alpha+\beta}$ and hence $J^{\alpha} J^{\beta}=J^{\alpha+\beta}$. (5) follows from (4), (3), (2) above. For if $J^{\alpha} f=0$ for some $\alpha$ in $\operatorname{Re} \alpha>0, J^{\alpha+r} f=0$ for all real numbers $r>0$. The principle of uniqueness for analytic functions implies that $J^{\alpha} f=0$ for all $\alpha$ in $\operatorname{Re} \alpha>0$. Since $\lim \left\{J^{r} f: r \rightarrow 0, r\right.$ is real $\}=f$ we have that $f=0$ and $J^{\alpha}$ is one-one.

Set $J^{1}=J$. To prove (6), we need verify the statement only for $0<\operatorname{Re} \alpha<1$. For if $\alpha=n+\beta$, we have $J^{\alpha}=J^{n} J^{\beta}$ from (4) above. If $\operatorname{Re} \alpha=n$, a positive integer, the conclusion (6) is proved in [6]. It is proved in [10] that $J^{n}=(J)^{n}$. So if we verify (6) when $0<\operatorname{Re} \beta<1, \mathrm{~K}-6$ of $\S 2 \mathrm{c}$ implies that $J^{\alpha}=(J)^{\alpha}$. Note that $J=(1+T)^{-1}$ when 
$P_{t}=\exp (-t T)$. So $J f=\int_{0}^{\infty} e^{-t} P_{t} f d t$. Then

$$
\begin{aligned}
J^{\alpha}(f) & =\Gamma(\alpha)^{-1} \int_{0}^{\infty} u^{\alpha-1} e^{-u} P_{u}(f) d u \\
& =\Gamma(\alpha)^{-1} \Gamma(1-\alpha)^{-1} \int_{0}^{\infty}\left(\int_{0}^{\infty} t^{-\alpha} e^{-u} e^{-t u} d t\right) P_{u} f d u \\
& =\Gamma(\alpha)^{-1} \Gamma(1-\alpha)^{-1} \int_{0}^{\infty}\left(\int_{1}^{\infty}(t-1)^{-\alpha} e^{-t u} d t\right) P_{u} f d u \\
& =\Gamma(\alpha)^{-1} \Gamma(1-\alpha)^{-1} \int_{1}^{\infty}(t-1)^{-\alpha} \int_{0}^{\infty} e^{-u t} P_{u} f d u d t \\
& =\Gamma(\alpha)^{-1} \Gamma(1-\alpha)^{-1} \int_{1}^{\infty}(t-1)^{-\alpha}(t+T)^{-1}(f) d t \\
& =\Gamma(\alpha)^{-1} \Gamma(1-\alpha)^{-1} \int_{0}^{\infty} v^{-\alpha}(v+1+T)^{-1} f d v
\end{aligned}
$$

Since $(v+1+T)^{-1}=J(v J+1)^{-1}$, set $v=x^{-1}$ to get by $\mathrm{K}-1$ that

$$
J^{\alpha}(f)=\frac{\sin \pi \alpha}{\pi} \int_{0}^{\infty} x^{\alpha-1} J(x+J)^{-1} f d x=(J)^{\alpha}(f) .
$$

(7) follows from Theorem 6.4 of [10] since by this theorem $D\left((1+T)^{\alpha}\right) \subset R\left(J^{\alpha}\right)$; $D\left((1+T)^{\alpha}\right)$ is dense in $L_{p}(H)$.

Parts (8) and (9) of Theorem 3 require a lengthy development; this is given in [6].

Definition. $L_{p}^{\alpha}(H)$ is the range of $J^{\alpha}$ acting on $L_{p}(H), \operatorname{Re} \alpha>0$. If $g \in L_{p}^{\alpha}(H)$, $\|g\|_{p, \alpha}=\|f\|_{p}$ when $g=J^{\alpha} f$.

REMARK. In [6] a more extensive list of the properties of the Bessel potential and its relationship to fractional powers is given. Because of Properties (8), (9) of Theorem 3 (proved in [6]), $L_{p}^{\alpha}(H)=L_{p}^{\mathrm{Re} \alpha}(H)$ with equivalence of the norms.

Since the translation operators $T_{t B y}, t>0$, form a bounded semigroup, $\left(-A_{y}\right)^{\alpha}$, $\operatorname{Re} \alpha>0$, can be written as in K-1 or K-3 of $\S 2 \mathrm{c}$. In order to be able to succinctly represent the hypersingular integrals of $\S 4$, we need to study the composition $\left(-A_{h}\right)^{\alpha} J^{\beta}$. To do this we need

LemMa 1. If $f$ is in $L_{p}(H), 1<p<\infty$, and if $\varphi(t)$ is the Fourier transform of a bounded, even, Borel measure $\mu$ on the real line, then

$$
\Phi_{A}(f)=\lim _{\varepsilon \rightarrow 0} \int_{|t|>\varepsilon} T_{t y} f \varphi\left(t A^{-1}\right) d t / t
$$

satisfies $\left\|\Phi_{A}(f)\right\|_{p} \leqq N(p)\|\mu\|\|f\|_{p}$ where the constant $N(p)$ is independent of $A$ and $y$ in $H$.

Proof. First set

$$
\left(T_{A} f\right)(x)=\lim _{\varepsilon \rightarrow 0} \int_{|t|>\varepsilon} f(x-t) \varphi\left(t A^{-1}\right) d t / t
$$


for $f$ in $L_{p}[(-\infty, \infty), d x]$. Then for smooth $f$ with compact support

$$
\left(T_{A} f\right)(x)=\int_{-\infty}^{\infty} e^{i u x} \lim _{\varepsilon \rightarrow 0} \int_{|y|>\varepsilon} f(x-y) e^{i u(y-x)} d y / y d \mu_{A}(u)
$$

where $\mu_{A}(E)=\mu(A E)$ for Borel sets $E$ in the real line. By the M. Reisz theorem on the Hilbert transform, $\left\|T_{A} f\right\|_{p} \leqq N(p)\|\mu\|\|f\|_{p}$, since $\|\mu\|=\left\|\mu_{A}\right\|$ for all $|A|>0$; $N(p)$ depends only on $p$.

Let $f$ be a bounded continuous tame function on $H$ which is based in the finitedimensional subspace $E$ of $H$; dimension of $E=k$. Since the normal distribution on $H$ is rotationally invariant, let $K$ be the span of $E$ and $y$ and let $e_{1}, \ldots, e_{k+1}$ be an orthonormal basis for $K$ with $e_{1}=\omega=y\|y\|^{-1}$. Then

$$
\left\|\int_{\delta<|t|<\rho} T_{t y} f \varphi\left(t A^{-1}\right) d t / t\right\|_{p}^{p}=\int_{K}\left|\int_{\delta|| y\|\leqq|t| \leqq \rho\| y \|} g(x-t \omega) \varphi\left(t\|y\|^{-1} A^{-1}\right) d t / t\right|^{p} d n(x)
$$

where $g$ is the restriction of $f$ to $K$ and where $D_{p}(x, t \omega)=\exp \left[(x, t \omega) / p-t^{2} / 2 p\right]$. If we write the integral over $K$ as an iterated integral and write the first integral as

$$
\begin{array}{r}
M \int_{-\infty}^{\infty} \mid \int_{\delta\||y| \leq|t|<\rho\| y \|} g\left(x_{1}-t, x_{2}, \ldots, x_{k+1}\right) \\
\cdot \exp \left[\frac{-\left(x_{1}-t\right)^{2}}{2 p}\right] \varphi\left(t\|y\|^{-1} A^{-1}\right) d t /\left.t\right|^{p} d x_{1},
\end{array}
$$

it follows from the discussion of $T_{A}$ over $(-\infty, \infty)$ in the first paragraph of this proof and from the dominated convergence theorem that $\left\|\Phi_{A}(f)\right\|_{p} \leqq N(p)\|\mu\|\|f\|_{p}$, the desired conclusion.

THEOREM 4. If $0<\operatorname{Re} \beta \leqq \operatorname{Re} \alpha,\left(-A_{h}\right)^{\beta} J^{\alpha}$ is a bounded operator on $L_{p}(H)$ for $1<p<\infty$ with $\left\|\left(-A_{h}\right)^{\beta} J^{\alpha}\right\| \leqq N(\alpha, \beta, p)\|h\|^{\mathrm{Re} \beta}$.

Proof. By P-3 of $\S 2 b$,

$$
A_{h} H_{t^{2}}(f)=t^{-1} \int_{H_{B}} T_{t B y} f C_{h}(1)(y) d n_{1}(y)
$$

where $C_{h}$ is the infinitesimal generator of $T_{s h}$ acting on $L_{1}\left(H, n_{1}\right)$. Since $A_{h}$ is a closed operator,

$$
A_{h} J(f)=\int_{0^{+}}^{\infty} \int_{H_{B}} T_{t B y} f C_{h}(1)(y) d n_{1}(y) \varphi(t) d t / t
$$

where $\varphi(t)=t^{-2} \int_{0}^{\infty} z \exp \left(-z^{2} t^{-2}\right) e^{-z} d z, t>0$. Since $C_{h}(1)(y)$ is a homogeneous polynomial of degree 1 in $y$, this last integral may be written as

$$
A_{h} J(f)=\frac{1}{2} \lim _{\varepsilon \rightarrow 0} \int_{|t|>\varepsilon} \int_{H_{B}} T_{t B y} f C_{h}(1)(y) d n_{1}(y) \varphi(t) d t / t
$$


when $\varphi(t)=\varphi(-t)$ for negative $t$. By Minkowski's integral inequality,

$$
\begin{aligned}
\left\|A_{h} J f\right\|_{p} & \leqq M \int_{H_{B}}\left\|\Phi_{\|B y\|}(f)\right\|_{p}\left|C_{h}(1)(y)\right| d n_{1}(y) \\
& \leqq N(p)\|\mu\|\|f\|_{p}
\end{aligned}
$$

by Lemma 1, if $\varphi(t)$ is the Fourier transform of a finite even Borel measure $\mu$ on the real line. Note that $\varphi(t)$ is even by definition and that on $t>0$

$$
\varphi(t)=\int_{0}^{\infty} z \exp \left[-z^{2}-t z\right] d z
$$

so that $\varphi^{\prime}(t) \leqq 0$ and $\varphi^{\prime \prime}(t)>0$. Thus Polya's criterion [3, p. 169] guarantees that $\varphi(t)$ is the Fourier transform of a finite even Borel measure on the line.

To prove that $\left(-A_{h}\right)^{\alpha} J^{\alpha}, 0<\operatorname{Re} \alpha<1$, is a bounded operator on $L_{p}(H), 1<p<\infty$, we consider the integral definition of $J^{\alpha}$ in terms of $H_{t}$. Begin by changing variables to replace $t$ by $t^{2}$. Then by arguing as above (or as in [5]), we get that

$$
\left(-A_{h}\right)^{\alpha} H_{t^{2}}(f)=t^{-\alpha} \int_{H_{B}} T_{t B y} f C_{h}^{\alpha}(1)(y) d n_{1}(y)
$$

where

$$
C_{h}^{\alpha}(1)(y)=\Gamma(-\alpha)^{-1} \int_{0^{+}}^{\infty}\left[\exp \left(2 t\langle y, h\rangle-t^{2}\|h\|^{2}\right)-1\right] t^{-\alpha-1} d t .
$$

Thus $C_{h}^{\alpha}(1)(y)$ is a tame function on $H$ based on the line through $h$. If $0<\varepsilon<R<\infty$, each of the functions

$$
E_{\varepsilon, R}(y, h)=\int_{\varepsilon}^{R}\left[\exp \left(2 t\langle y, h\rangle-t^{2}\|h\|^{2}\right)-1\right] t^{-\alpha-1} d t
$$

has integral zero. By Minkowski’s integral inequality,

$$
\left\|E_{R, \rho}(y, h)\right\|_{1} \leqq N(\alpha, h)\left(\rho^{-\mathrm{R} \theta \alpha}+R^{-\mathrm{Re} \alpha}\right)
$$

for large $\rho$ and $R$, and integration by parts shows that

$$
\left\|E_{\varepsilon, \delta}(y, h)\right\|_{1} \leqq N(\alpha, h)\left(\varepsilon^{1-\operatorname{Re} \alpha}+\delta^{1-\mathrm{R} e \alpha}\right) .
$$

Thus $C_{h}^{\alpha}(1)(y) \in L_{1}\left(H, n_{1}\right)$ and $\int_{H} C_{h}^{\alpha}(1)(y) d n_{1}(y)=0$. To apply the techniques of [4] to prove the boundedness of

$$
\left(-A_{h}\right)^{\alpha} J^{\alpha}(f)=M(\alpha) \int_{0}^{\infty} u^{\alpha} e^{-u^{2}}\left[\int_{0+}^{\infty} \int_{H_{B}} T_{t B y} f C_{h}^{\alpha}(1)(y) d n_{1}(y) e^{-t u} d t / t\right] d u,
$$

set $\varphi(t)=e^{-|t| u}$ and verify that the even part of $C_{h}^{\alpha}(1)(y)$ is in $L \log ^{+} L\left(H, n_{1}\right)$. This definition of $\varphi(t)$ allows us to use Lemma 1 when $C_{h}^{\alpha}(1)(y)$ is replaced by its odd part and to use Lemma 1 when the techniques of the proof of Theorem 8 of [4] are applied to the even part of $C_{h}^{\alpha}(1)(y)$. 
Set $C_{h}^{\alpha}(1)(y)=K(\alpha)\|h\|^{\alpha} G(y)$ where $G(y)$ is a tame function based on the line through $h$; so it suffices to prove that

$$
G(x)=\int_{0^{+}}^{\infty}\left[\exp \left(2 t x-t^{2}\right)-1\right] t^{-\alpha-1} d t
$$

has even part in $L \log ^{+} L\left((-\infty, \infty)\right.$, exp $\left.\left(-x^{2}\right) d x\right)$. Integrate by parts to write

$$
G(x)=K(\alpha) \int_{0^{+}}^{\infty}(x-t) \exp \left(2 t x-t^{2}\right) t^{-\alpha} d t
$$

Expand $\exp (2 t x)$ in a Taylor series about 0 and integrate term-by-term. After some calculations with identities in the $\Gamma$-function we get that

$$
G(x)=K(\alpha) \sum_{k=0}^{\infty} \frac{2^{k} \Gamma((k-\alpha) / 2)}{k !} x^{k} .
$$

The ratio test shows that this series converges uniformly on the compact subsets of the line. Let $L(x)=\frac{1}{2}(G(x)+G(-x))$;

$$
L(x)=K(\alpha) \sum_{m=0}^{\infty} \frac{2^{2 m} \Gamma(m-\alpha / 2)}{(2 m) !} x^{2 m} .
$$

By using the fact that $(\pi)^{1 / 2} \Gamma(2 z)=2^{2 z-1} \Gamma(z) \Gamma\left(z+\frac{1}{2}\right)$, we see that

$$
2^{2 m} \Gamma(m-\alpha / 2) \Gamma(2 m+1)^{-1}=K \Gamma(m-\alpha / 2) \Gamma\left(m+\frac{1}{2}\right)^{-1} \Gamma(m+1)^{-1} .
$$

Since $\operatorname{Re} \alpha<1,|\Gamma(m-\alpha / 2)| \Gamma\left(m+\frac{1}{2}\right)^{-1} \leqq \Gamma(m-(\operatorname{Re} \alpha) / 2) \Gamma\left(m+\frac{1}{2}\right)^{-1} \leqq 1$, and $|L(x)|$ $\leqq N(\alpha) \exp \left(x^{2}\right)$. Thus $\log ^{+}|L(x)| \leqq N(\alpha)+x^{2}$, and since

$$
\int_{-\infty}^{\infty}|L(x)| \exp \left(-x^{2}\right) d x<\infty
$$

it suffices to show that $\int_{-\infty}^{\infty} x^{2}|L(x)| \exp \left(-x^{2}\right) d x<\infty$. By integrating the above series for $L$ term-by-term with respect to $x^{2} \exp \left(-x^{2}\right)$ and applying the ratio test to the resulting series, one sees that $L(x)$ is in $L \log ^{+} L$.

In order to make use of Theorem 5 of [1] we also need to verify that $\int_{-\infty}^{\infty} \log ^{+}|x||L(x)| \exp \left(-x^{2}\right) d x<\infty$. But this follows from the fact that $\log ^{+}|x|$ $\leqq \varepsilon^{-1}|x|^{\varepsilon}$ for $\varepsilon>0$ when $|x| \geqq 0$ and a calculation similar to that above for $\int_{-\infty}^{\infty}|x|^{\varepsilon}|L(x)| \exp \left(-x^{2}\right) d x$. This last integral is seen to be finite for all sufficiently small $\varepsilon>0$ since $\operatorname{Re} \alpha<1$.

Now write $C_{h}^{\alpha}(1)(y)=K(\alpha)(L(y)+M(y))\|h\|^{\alpha}$ where $L(y)=\frac{1}{2}(G(y)+G(-y))$ and where $M(y)=\frac{1}{2}(G(y)-G(-y))$ so that $\left(-A_{h}\right)^{\alpha} J^{\alpha}(f)=T_{L}(f)+T_{M}(f)$ where $T_{L}$ and $T_{M}$ are integral operators of the form of $\left(-A_{h}\right)^{\alpha} J^{\alpha}$ with $C_{h}^{\alpha}(1)(y)$ replaced by $L(y)$ and $M(y)$ respectively. Since $M(y)$ is an odd function, $T_{M}$ can be written as an integral of an operator of the type in Lemma 1 , so that $T_{M}$ is a bounded operator on $L_{p}(H)$ if $1<p<\infty$. For $T_{L}$ we proceed as in the even kernel case in [4]. Let $K_{h}(f)=\pi^{-1} P \int_{-\infty}^{\infty} T_{v B h}(f) d v / v$, the principal value integral. By Lemma $1, K_{h}$ is a 
bounded operator on $L_{p}(H) ; K_{h}^{2}(f)=-f$. This last fact follows from the observation that $K_{h}$ is just the Hilbert transform in the direction $B h$. Consider $K_{h} T_{L}$. Direct computation shows that $K_{h} T_{L}(f)$ is an integral with respect to $u$ of

$$
\Delta_{h}(f)=\int_{0^{+}}^{\infty} \int_{H_{B}} T_{t u B y} f K_{h}^{\prime} L(y) d n_{1}(y) \varphi(t) d t / t
$$

where $K_{h}^{\prime} L$ is the function $P \int_{-\infty}^{\infty} S_{u h} L d u / u$ where $y \rightarrow S_{y}$ is the regular representation of the additive group of $H$ acting on $L_{1}\left(H, n_{1}\right)$. Since $L(y)$ has integral zero with respect to $n_{1}$ on $H_{B}$, since $L(y)$ is a tame function based on the line through $h$, and since $L \in L \log ^{+} L\left(H, n_{1}\right)$ with $|\langle x, h\rangle||L(x)| \in L_{1}\left(H, n_{1}\right)$, by Theorem 5 of [1], $K_{h}^{\prime}(L)$ is an odd function in $L_{1}\left(H, n_{1}\right)$. Thus

$$
\left\|T_{L}(f)\right\|_{p}=\left\|K_{h} K_{h} T_{L}(f)\right\|_{p} \leqq N(p)\left\|K_{h} T_{L}(f)\right\|_{p} \leqq K(p, \alpha)\|h\|^{\operatorname{Re} \alpha}\|f\|_{p} .
$$

The desired conclusion regarding $\left(-A_{h}\right)^{\alpha} J^{\alpha}$ follows when $0<\operatorname{Re} \alpha<1$.

If $\operatorname{Re} \alpha>1$, use K-6. If $\operatorname{Re} \beta<\operatorname{Re} \alpha$, we have $\left(-A_{h}\right)^{\beta} J^{\alpha}=\left(-A_{h}\right)^{\beta} J^{\beta} J^{\alpha-\beta}$ by part 4 of Theorem 3 with $\left(-A_{h}\right)^{\beta} J^{\beta}$ and $J^{\alpha-\beta}$ bounded on $L_{p}(H)$, so that $\left(-A_{h}\right)^{\beta} J^{\alpha}$ is bounded on $L_{p}(H)$ when $\operatorname{Re} \beta \leqq \operatorname{Re} \alpha$. This completes the proof of Theorem 4 .

In the next theorem we look at the most elementary and one of the most interesting hypersingular integral operators. This operator was st.idied by E. M. Stein in [17], [18]. Let $P_{t}(f)=\exp (-t T) f$. Then

$$
T^{\alpha}(f)=\Gamma(-\alpha)^{-1} \int_{0}^{\infty}\left(P_{t} f-f\right) t^{-\alpha-1} d t
$$

for $0<\operatorname{Re} \alpha<1$. We shall examine the action of $T^{\alpha} J^{\alpha}$ on $L_{p}(H)$ for $\operatorname{Re} \alpha>0$.

THEOREM 5. If $0<\operatorname{Re} \alpha<1$,

$$
T^{\alpha} J^{\alpha}(f)=f-\frac{\sin \pi \alpha}{\pi} \int_{0}^{1} J_{u}(f) u^{\alpha}(1-u)^{-\alpha} d u
$$

where $J_{u}(f)=\int_{0}^{\infty} P_{t}(f) e^{-t u} d t . T^{\alpha} J^{\alpha}(f)$ is given by convolution with the measure

$$
\nu_{\alpha}(E)=\delta_{0}(E)-\frac{\sin \pi \alpha}{\pi} \int_{0}^{1} j_{u}(E) u^{\alpha}(1-u)^{-\alpha} d u
$$

where $j_{u}(E)=\int_{0}^{\infty} p_{t}(E) e^{-t u} d t$ and $P_{t}(f)=\int_{H} T_{y} f d p_{t}(y)$, and where $\delta_{0}(E)$ is the probability measure concentrated at the origin in $H . T^{1} J^{1}$ is given by convolution with the measure $\nu_{1}(E)=\delta_{0}(E)-j_{1}(E)$ and $T^{\alpha} J^{\alpha}$ is given by convolution with the measure $\nu_{\alpha}=\nu_{\beta} * \nu_{n}, \nu_{n}=\nu_{1} * \nu_{1} * \cdots * \nu_{1}$ (n times) if $\alpha=n+\beta$ with $\operatorname{Re} \beta<1$. If $\operatorname{Re} \alpha=n$, an integer, $v_{\alpha}=\nu_{\alpha-1 / 2} * v_{1 / 2}$.

Proof. Notice first that

$$
T J(f)=\int_{0}^{\infty} T P_{t}(f) e^{-t} d t=-\int_{0}^{\infty} \frac{\partial}{\partial t} P_{t}(f) e^{-t} d t=f-J f
$$


by integration by parts. Thus if $\delta_{0}$ is the Borel probability measure concentrated at the origin in $H$,

$$
T J(f)=\int_{H} T_{y} f d \nu_{1}(y), \quad \nu_{1}(y)=\delta_{0}(E)-j_{1}(E) .
$$

For any positive integer $n, T^{n} J^{n}(f)=\int_{H} T_{y} f d \nu_{n}(y)$ where $\nu_{n}$ is the $n$-fold convolution of $\nu_{1}$.

For $0<\operatorname{Re} \alpha<1$,

$$
J^{\alpha}(f)=\Gamma(\alpha)^{-1} \int_{0}^{\infty} P_{t}(f) t^{\alpha-1} e^{-t} d t
$$

and

$$
T^{\alpha}(f)=\Gamma(-\alpha)^{-1} \int_{0}^{\infty}\left(P_{y} f-f\right) y^{-1-\alpha} d y .
$$

If $f \in D(T), \quad T^{\alpha}(f)=-\Gamma(1-\alpha)^{-1} \int_{0}^{\infty} P_{y}^{\prime}(f) y^{-\alpha} d y$ where $P_{y}^{\prime}(f)=\partial P_{y}(f) / \partial y$. Let $L(g(t))(x)$ be the Laplace transform of $g(t)$ at $x$. Then

$$
T^{\alpha} J^{\alpha}(f)=-\frac{\sin \pi \alpha}{\pi} \int_{1}^{\infty} L\left(t^{\alpha} T_{0}^{\alpha} P_{t}(f)\right)(x) d x,
$$

where $T_{0}^{\alpha}=\Gamma(-\alpha) T^{\alpha}$. Now

$$
\begin{aligned}
t^{\alpha} T_{0}^{\alpha}\left(P_{t} f\right) & =t^{\alpha} \int_{0+}^{\infty} \frac{\partial}{\partial y} P_{y+t}(f) y^{-\alpha} d y \\
& =\int_{0+}^{\infty} \frac{\partial}{\partial t} P_{y+t}(f) t^{\alpha} y^{-\alpha} d y=\int_{0^{+}}^{\infty} t P_{t}^{\prime} P_{t y}(f) y^{-\alpha} d y .
\end{aligned}
$$

But $P_{t}^{\prime} P_{t y}(f)=(y+1)^{-1} \partial P_{t(y+1)}(f) / \partial t$. Thus

$$
\begin{aligned}
L\left(t^{\alpha} T_{0}^{\alpha}\left(P_{t}(f)\right)\right)(x) & =\int_{0^{+}}^{\infty}(y+1)^{-1}\left(-\frac{\partial}{\partial x} x\right) L\left(P_{t(y+1)}(f)\right)(x) y^{-\alpha} d y \\
& =\int_{0^{+}}^{\infty}(y+1)^{-2}\left(-\frac{\partial}{\partial x} x\right) J_{x(y+1)^{-1}}(f) y^{-\alpha} d y
\end{aligned}
$$

where $J_{u}(f)=L\left(P_{t} f\right)(u)$. Since

$$
\left\|\left(-\frac{\partial}{\partial x} x\right) J_{x(y+1)^{-1}}(f)\right\| \leqq K(y+1) x^{-1}\|f\|_{p}
$$

we consider an interchange of integrals in $\int_{1}^{R} L\left(t^{\alpha} T_{0}^{\alpha}\left(P_{t}(f)\right)\right)(x) d x$.

$$
\int_{1}^{R}(y+1)^{-2}\left(-\frac{\partial}{\partial x} x\right) J_{x(y+1)^{-1}}(f) d x=(y+1)^{-2}\left[J_{(y+1)^{-1}}(f)-R J_{R(y+1)^{-1}}(f)\right] .
$$

Since $\left\|R J_{R(y+1)^{-1}}(f)\right\|_{p} \leqq(y+1) K\|f\|_{p}$, the dominated convergence theorem implies that

$$
T^{\alpha} J^{\alpha}(f)=-\frac{\sin \pi \alpha}{\pi} \int_{0^{+}}^{\infty}\left(J_{(1+y)^{-1}}(f)-(y+1) f\right) y^{-\alpha}(1+y)^{-2} d y
$$


Set $u=(y+1)^{-1}$ to get

$$
\begin{aligned}
T^{\alpha} J^{\alpha}(f) & =-\frac{\sin \pi \alpha}{\pi} \int_{0}^{1}\left(J_{u}(f)-u^{-1} f\right) u^{\alpha}(1-u)^{-\alpha} d u \\
& =-\frac{\sin \pi \alpha}{\pi} \int_{0}^{1} J_{u}(f) u^{\alpha}(1-u)^{-\alpha} d u+\frac{\sin \pi \alpha}{\pi} \int_{0}^{1} u^{\alpha-1}(1-u)^{-\alpha} d u f . \\
& =f-\frac{\sin \pi \alpha}{\pi} \int_{0}^{1} J_{u}(f) u^{\alpha}(1-u)^{-\alpha} d u .
\end{aligned}
$$

If $\operatorname{Re} \alpha>0$ and $\operatorname{Re} \alpha$ is not an integer, write $\alpha=n+\beta$ where $n$ is a positive integer and $0<\operatorname{Re} \beta<1$. Set

$$
\nu_{\beta}(E)=\delta_{0}(E)-\frac{\sin \pi \beta}{\pi} \int_{0}^{1} j_{u}(E) u^{\beta}(1-u)^{-\beta} d u
$$

for Borel sets $E$. Then $\nu_{\alpha}=\nu_{n} * \nu_{\beta}$ is the Borel measure such that $T^{\alpha} J^{\alpha}(f)$ $=\int_{H} T_{y}(f) d \nu_{\alpha}(y)$. If $\operatorname{Re} \alpha=n$, a positive integer, let $\gamma=\alpha-\frac{1}{2}$ and $\beta=\frac{1}{2}$. Then $\nu_{\alpha}=\nu_{\gamma} * \nu_{\beta}$, and $T^{\alpha} J^{\alpha}(f)=\int_{H} T_{y}(f) d \nu_{\alpha}(y)$. K-6 and the continuity of all of the operators in question justifies these last designations of $\nu_{\alpha}$.

REMARKS. 1. It is interesting to note that $T^{\alpha} J^{\alpha}$ is a bounded operator on $L_{1}(H)$. It will not be possible to make this claim for the hypersingular integral operators considered in $\$ 4$.

2. The calculation in the proof of Theorem 5 holds for any bounded strongly continuous semigroup $S_{t}$. Note that the proof of Theorem 5 amounts to calculating $A^{\alpha}(1+A)^{-\alpha}$ when $S_{t}=\exp (-t A)$. Komatsu $[10, \S 6]$ has made this general calculation using methods different from those in Theorem 5; see p. 309 of [10].

3. $L_{p}^{\alpha}(H)=L_{p}^{\mathrm{Re} \alpha}(H)$ with equivalent norms; see [6].

4. For $\alpha>0$ other useful forms of $J^{\alpha}$ exist and define equivalent norms on $L_{p}^{\alpha}(H)$. For instance $J_{2}^{\alpha}(f)=\Gamma(\alpha / 2)^{-1} \int_{0}^{\infty} H_{t}(f) t^{(\alpha-2) / 2} e^{-t} d t$. In the language of [10], $J^{\alpha}$ $=J_{1}^{\alpha}=(1+T)^{-\alpha}$ and $J_{2}=\left(1+T^{2}\right)^{-\alpha / 2}$. We have shown in [6] that $J^{\alpha}$ and $J_{2}^{\alpha}$ define equivalent norms on $L_{p}^{\alpha}(H)$ and that $L_{p}^{\alpha}(H)$ is equivalent to $D\left(T^{\alpha}\right)$ when this domain is equipped with the graph norm. [6] also contains a proof that $J_{1}^{\alpha}$ and $J_{2}^{\alpha}$ have strongly continuous boundary value groups $J_{1}^{i \gamma}$ and $J_{2}^{i \gamma}$ consisting of bounded operators on $L_{p}(H), 1<p<\infty$.

4. Hypersingular integrals. In this section we shall study the general class of hypersingular integral operators on $L_{p}^{\alpha}(H)$.

THEOREM 6. Let $\mu$ be a Borel measure (possibly unbounded) on $H$ such that $\int_{H}\|y\|^{\operatorname{Re} \alpha} d|\mu|(y)<\infty$. If $n<\operatorname{Re} \alpha<n+1, n$ a nonnegative integer, set

$$
G^{\alpha}(f)=\int_{0^{+}}^{\infty} \int_{H} R_{n}(f, y, t) d \mu(y) t^{-\alpha-1} d t
$$

where $R_{n}(f, y, t)=((n-1) !)^{-1} \int_{0}^{t}(t-u)^{n-1}\left(T_{u B y} A_{y}^{n} f-A_{y}^{n} f\right) d u$ if $n \geqq 1$ and $R_{0}(f, y, t)$ 
$=T_{t B y} f-f$ if $n=0$. Then

and

$$
G^{\alpha} J^{\alpha}(f)=M(\alpha) \int_{H}\left(-A_{y}\right)^{\alpha} J^{\alpha}(f) d \mu(y)
$$

$$
\left\|G^{\alpha} J^{\alpha}(f)\right\|_{p} \leqq N(\alpha, p) \int_{H}\|y\|^{\operatorname{Re} \alpha} d|\mu|(y)
$$

where $M(\alpha)$ and $N(\alpha, p)$ are constants which depend only on the parameters shown.

Proof. Set $\alpha=n+\beta, 0<\operatorname{Re} \beta<1$, and note that $t^{-n} R_{n}(f, y, t)=t^{-n} R_{n}(f, t y, 1)$. Also note that $A_{t y}^{n}=t^{n} A_{y}^{n}$ and

$$
\int_{0^{+}}^{\infty}\left(T_{t u B y} J^{\alpha}(f)-J^{\alpha}(f)\right) t^{-\beta-1} d t=M(\alpha) u^{\beta}\left(-A_{y}\right)^{\beta} J^{\alpha}(f)
$$

Since $\left|\int_{0}^{1}(1-u)^{n-1} u^{\beta} d u\right|<\infty$, we have that

$$
G^{\alpha} J^{\alpha}(f)=M(\alpha) \int_{H} A_{y}^{n}\left(-A_{y}\right)^{\beta} J^{\alpha}(f) d \mu(y) .
$$

By K-6, $G^{\alpha} J^{\alpha}$ has the desired form. By Theorem 4, $\left\|\left(-A_{y}\right)^{\alpha} J^{\alpha} f\right\|_{p} \leqq N(\alpha, p)\|y\|^{\text {Re } \alpha}$, so that by Minkowski's integral inequality we get the desired estimate for $\left\|G^{\alpha} J^{\alpha}(f)\right\|_{p}$.

REMARK. The constant $M(\alpha)$ in the first conclusion of Theorem 6 is $M(\alpha)$ $=\Gamma(n)^{-1} \Gamma(-\beta) B(n, \beta+1)(-1)^{n}=(-1) \Gamma(\alpha+1)^{-1} \Gamma(1-\alpha) \Gamma(\alpha)$.

In the case when $\operatorname{Re} \alpha=n$, a nonnegative integer, the desired theorem is more complicated. We present a theorem and a remark regarding this situation. We begin with

Lemma 2. Let $h$ be a unit vector in $H$ and $\gamma \neq 0$ be a real number. Then

$$
T(f)=\lim _{\varepsilon \rightarrow 0+}\left[\int_{\varepsilon}^{\infty} T_{t h} f t^{-i \gamma-1} d t-\frac{\varepsilon^{-i \gamma} f}{i \gamma}\right]
$$

exists as a bounded operator on $L_{p}(H)$ and $\|T\|_{p} \leqq K p q\left(|\gamma|^{2}+1\right)|\gamma|^{-1}$.

Proof. By Theorem 8 of [14], if

$$
U_{\varepsilon}(f)(x)=\int_{\varepsilon}^{\infty} f(x-t) t^{-i \gamma-1} d t-\frac{\varepsilon^{-i \gamma} f(x)}{i \gamma},
$$

the $U_{\varepsilon}$ are uniformly bounded as operators on $L_{p}((-\infty, \infty), d x)$ and as $\varepsilon \rightarrow 0^{+}$, $U_{\varepsilon}$ converges strongly to a bounded operator on $L_{p}, 1<p<\infty$. Let $f$ be a bounded tame function on $H$ which is based in a finite-dimensional subspace $F$ of $H$. Let $K$ be the span of $F$ and $h$ and let $g$ be the restriction of $f$ to $K$. By arguing as in the proof of Theorem 4, we show that the $T_{\varepsilon}$ converge strongly to a bounded operator $T$ on $L_{p}(H)$. The $T_{\varepsilon}$ are uniformly bounded and $\|T\|_{p} \leqq K p q\left(|\gamma|^{2}+1\right)|\gamma|^{-1}$, where $q$ is conjugate to $p$. 
THEOREM 7. If $\gamma \neq 0$ is a real number, $n$ is a nonnegative integer, and $\mu$ is a Borel measure (not necessarily bounded) on $H$ such that $\int_{H} p_{n}(y) d \mu(y)=0$ when $p_{n}(y)$ is a homogeneous polynomial of degree $n$ on $H$ and $\mu$ is such that $\int_{H}\|y\|^{n} d|\mu|(y)<\infty$, then $G^{\alpha} J^{\alpha}, \alpha=n+i \gamma$, is a bounded operator on $L_{p}(H)$ with

$$
\left\|G^{\alpha} J^{\alpha}\right\| \leqq N(n, \gamma, p) \int_{H}\|y\|^{n} d|\mu|(y)
$$

Proof. Since $\int_{H} p_{n}(y) d \mu(y)=0, \int_{H} A_{y}^{n} J^{\alpha} f d \mu(y)=0$, and $\int_{H} R_{n}(f, y, t) d \mu(y)$ $=\int_{H} R_{n-1}(f, y, t) d \mu(y)$. Write

$$
R_{n-1}(f, y, t)=\Gamma(n)^{-1} \int_{0}^{t}(t-u)^{n-1} T_{u B y} A_{y}^{n} f d u .
$$

Then $t^{-n} R_{n-1}(f, y, t)=t^{-n} R_{n-1}(f, t y, 1), A_{t y}^{n}=t^{n} A_{y}^{n}$, and

$$
\begin{aligned}
& G^{\alpha} J^{\alpha}(f)= \int_{H} \int_{0+}^{\infty} t^{-n} R_{n-1}\left(J^{\alpha} f, t y, 1\right) t^{-i \gamma-1} d t d \mu(y) \\
&=K(n) \int_{H} \int_{0}^{1} \lim _{\varepsilon \rightarrow 0}\left[\int_{\varepsilon u\|B y\|}^{1}\left[T_{t h} A_{y}^{n} J^{\alpha} f-A_{y}^{n} J^{\alpha} f\right] t^{-i \gamma-1} d t-\frac{A_{y}^{n} J^{\alpha} f}{i \gamma}\right. \\
&\left.\quad+\int_{1}^{\infty} T_{t h} A_{y}^{n} J^{\alpha} f t^{-i \gamma-1} d t\right](1-u)^{n-1} u^{i \gamma} d u\|B y\|^{i \gamma} d \mu(y),
\end{aligned}
$$

where $h=B y\|B y\|^{-1}$. The inner most integral is

$$
\int_{\varepsilon u\|B y\|}^{\infty} T_{t h} A_{y}^{n} J^{\alpha} f t^{-i \gamma-1} d t-\frac{(\varepsilon\|B y\| u)^{-i \gamma}}{i \gamma} A_{y}^{n} J^{\alpha} f .
$$

By Corollary 4.1 of [6], $J^{\alpha}=J^{n} J^{i \gamma}$ and by Theorem 4 of [6], $J^{i \gamma}$ is a bounded operator on $L_{p}(H), 1<p<\infty$. So by Lemma 2 and the dominated convergence theorem, $G^{\alpha} J^{\alpha}$ is a bounded operator on $L_{p}(H)$ with $\left\|G^{\alpha} J^{\alpha}\right\| \leqq N(n, \gamma, p) \int_{H}\|y\|^{n} d|\mu|(y)$.

REMARKS. 1. When $\alpha=n$, a positive integer,

$$
G^{n} J^{n}(f)=M(n) \int_{0^{+}}^{\infty} \int_{H}\left[T_{t B y} A_{y}^{n} J^{n} f-A_{y}^{n} J^{n} f\right] d \mu(y) d t / t .
$$

If we assume that $\int_{H} p_{n}(y) d \mu(y)=0$ when $p_{n}(y)$ is a homogeneous polynomial of degree $n$ in $y$ and if we assume that $\int_{H}\|y\|^{n} d \mu(y)<\infty$, then $\int_{H} A_{y}^{n} J^{n} f d \mu(y)=0$ and $G^{n} J^{n}$ is a kind of Calderon-Zygmund singular integral operator on $L_{p}(H)$; see [4]. When $\alpha=0, G^{n} J^{n}$ is precisely the singular integral operator studied in [4]. We may write $\mu=\mu_{0}+\mu_{e}$ where $\mu_{0}(E)=\frac{1}{2}\left(\mu(E)-(-1)^{n} \mu(-E)\right)$ and $\mu_{e}(E)$ $=\frac{1}{2}\left(\mu(E)+(-1)^{n} \mu(-E)\right)$. Then $\mu_{0}(-E)=(-1)^{n+1} \mu_{0}(E)$ and $\mu_{e}(-E)=(-1)^{n} \mu_{e}(E)$; furthermore, $G^{n} J^{n}(f)=T_{0}(f)+T_{e}(f)$ where $T_{0}$ and $T_{e}$ are operators of the same form as $G^{n} J^{n}$ but have $\mu$ replaced by $\mu_{0}$ and $\mu_{e}$ respectively. Because

$$
\int_{H} T_{t B y} A_{y}^{n} J^{n}(f) d \mu_{0}(y)=-\int_{H} T_{-t B y} A_{y}^{n} J^{n}(f) d \mu_{0}(y)
$$


$T_{0}$ can be shown to be a bounded operator by using the M. Riesz theorem on the Hilbert transform as in the first paragraph of the proof of Theorem 4 above or as in the proof of Theorem 7 of [4]. If the measure $\mu_{e}$ is concentrated on a finitedimensional subspace $K$ of $H$,

$$
\int_{H} T_{t B y} A_{y}^{n} J^{n}(f) d \mu_{e}(y)=\int_{K} T_{t B x} A_{x}^{n} J^{n}(f) d \nu(x)
$$

where $\nu$ denotes the restriction of $\mu_{e}$ to $K$. Let $e_{1}, \ldots, e_{k}$ be an orthonormal basis for $K$ and let $R_{1}, \ldots, R_{k}$ be the Riesz operators used in the proof of Theorem 8 of [4]. Write $A_{x}^{n} J^{n}(f)=\sum_{|a|=n} x^{a} B_{a} A^{a} J^{n}(f)$ where $A^{a}=A_{1}^{a_{1}} \cdots A_{k}^{a_{k}}, a=\left(a_{1}, \ldots, a_{k}\right), B_{a}$ are constants and the $A_{i}$ are infinitesimal generators of the $T_{t B e_{i}}, i=1, \ldots, k$. Then $\int_{H} x^{a} d v(x)=0$. If we assume that $\nu$ is absolutely continuous with respect to the normal distribution with variance parameter 1 on $K$, and if $d \nu(x) / d n=\Omega(x)$ satisfies $x^{a} \Omega(x) \in L \log ^{+} L(K, n)$ for each multi-index $a$ with $|a|=n$, then the argument used in the proof of Theorem 8 of [4] can be used to prove that each of the

$$
\int_{0+} \int_{k} T_{t B x} A^{a} J^{n}(f) x^{a} \Omega d n(x) d t / t
$$

is bounded on $L_{p}(H)$; so that $T_{e}$ is bounded on $L_{p}(H)$.

2. In [6] we defined $J^{i \gamma}, \gamma$ real, and showed that the $J^{i \gamma}$ form a strongly continuous group of bounded operators on $L_{p}(H)$. Furthermore, the $J^{i \gamma}$ of [6] are suitable for boundary values of the analytic semigroup $J^{\alpha}, \operatorname{Re} \alpha>0$. In a paper yet to be published, we have shown that if $h \in H,\left(A_{h}\right)^{i \gamma}$ is a strongly continuous group of bounded operators on $L_{p}(H)$. These results suggest that when $\operatorname{Re} \alpha=n$, a positive integer, $\operatorname{Im} \alpha=i \gamma \neq 0$, and when $\int_{H} p_{n}(y) d \mu(y)=0$, then

$$
G^{\alpha} J^{\alpha}(f)=M(\alpha) \int_{H} A_{y}^{\alpha} J^{\alpha}(f) d \mu(y) .
$$

Since $A_{y}^{\alpha}(g)$ is an analytic function of $\alpha$ for a dense set of $g$ in $L_{p}(H)$, and since $M(\alpha)=M^{\prime}(\alpha)(\alpha-n)^{-1}$ where $M^{\prime}(\alpha)$ is a bounded function of $\alpha$ in a neighborhood of $n$ in the real line, we might ask whether

$$
G^{n} J^{n}(f)=\left.M(n) \int_{H} \frac{\partial}{\partial \alpha}\left(A_{y}^{\alpha} J^{\alpha} f\right)\right|_{\alpha=n} d \mu(y) .
$$

These topics require a lengthy development and will be treated in a sequel on singular integrals and fractional powers of operators; see [22].

\section{REFERENCES}

1. A. P. Calderón and A. Zygmund, On the existence of certain singular integrals, Acta Math. 88 (1952), 85-139. MR 14, 637.

2. A. P. Calderón, Lebesgue spaces of differentiable functions and distributions, Proc. Sympos. Pure Math., vol. 4, Amer. Math. Soc., Providence, R. I., 1961, pp. 33-49. MR 26 \#603.

3. K. L. Chung, A course in probability theory, Harcourt Brace \& World, New York, 1968. MR 37 \#4842. 
4. M. J. Fisher, Singular integral operators over a Hilbert space, Trans. Amer. Math. Soc. 131 (1968), 437-465. MR 36 \#6987.

5. - Harmonic functions and their conjugates on Hilbert space, Trans. Amer. Math. Soc. 137 (1969), 387-405.

6. - Fractional powers of operators and Bessel potentials on Hilbert space, Studia Math. (to appear).

7. L. Gross, Integration and nonlinear transformations in Hilbert space, Trans. Amer. Math. Soc. 94 (1960), 404-440. MR 22 \#2883.

8. - Harmonic analysis on Hilbert space, Mem. Amer. Math. Soc. No. 46 (1963). MR 28 \#4304.

9. - Abstract Wiener spaces, Proc. Fifth Berkeley Sympos. Math. Statist and Probability (Berkeley, Calif., 1965/66), vol. II : Contributions to Probability Theory, part 1, Univ. of California Press, Berkeley, Calif., 1967, pp. 31-42. MR 35 \#3027.

10. H. Komatsu, Fractional powers of operators, Pacific J. Math. 19 (1966), 285-346. MR 34 \#1862.

11. - Fractional powers of operators. II. Interpolation spaces, Pacific J. Math. 21 (1967), 89-111. MR 34 \#6533.

12. — Fractional powers of operators. III. Negative powers. J. Math. Soc. Japan 21 (1969), 205-220. MR 39 \#3340.

13. - Fractional powers of operators. IV. Potential operators, J. Math. Soc. Japan 21 (1969), 221-228. MR 39 \#3341.

14. B. Muckenhoupt, On certain singular integrals, Pacific J. Math. 10 (1960), 239-261. MR 22 \#3948.

15. I. E. Segal, Tensor algebras over Hilbert spaces. I, Trans. Amer. Math. Soc. 81 (1956), 106-134. MR 17, 880.

16. - Distributions in Hilbert space and canonical systems of operators, Trans. Amer. Math. Soc. 88 (1958), 12-41. MR 21 \#1545.

17. E. M. Stein, The characterization of functions arising as potentials, Bull. Amer. Math. Soc. 67 (1961), 102-104. MR 23 \#A1051.

18. - The characterization of functions arising as potentials. II, Bull. Amer. Math. Soc. 68 (1962), 577-582. MR 26 \#547.

19. R. L. Wheeden, On hypersingular integrals and Lebesgue spaces of differentiable functions, Trans. Amer. Math. Soc. 134 (1968), 421-435. MR 38 \#575.

20. - On hypersingular integrals and Lebesgue spaces of differentiable functions. II, Trans. Amer. Math. Soc. 139 (1969), 37-53. MR 39 \#2028.

21. K. Yosida, Functional analysis, Die Grundlehren der math. Wissenschaften, Band 123, Academic Press, New York; Springer-Verlag, Berlin, 1965. MR 31 \#5054.

22. M. J. Fisher, Purely imaginary powers of certain differential operators, Amer. J. Math. (to appear).

Johns Hopkins UNiversity, Baltimore, MARyland 21218

University of Montana, Missoula, Montana 59801 